
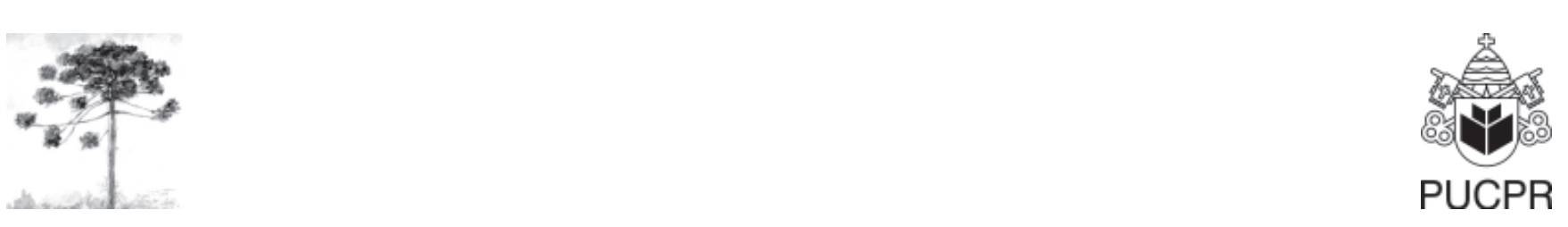

\title{
INDICADOR HIGIÊNICO-PARASITÁRIO EM MANIPULADORES DE ALIMENTOS EM MORRINHOS, GO
}

\author{
Hygienic parasitic indicators in food handlers in Morrinhos, GO
}

\author{
Roberta Marques Reis ${ }^{[1]}$, Lílian Carla Carneiro ${ }^{[2]}$ \\ ${ }^{[1]}$ Acadêmica da Universidade Estadual de Goiás (UEG), Goiás, GO - Brasil. \\ ${ }^{[2]}$ Professora da Universidade Estadual de Goiás (UEG), Goiás, GO - Brasil, e-mail: lilian.carneiro@ueg.br
}

\section{Resumo}

As doenças veiculadas pelos alimentos representam sério problema de saúde pública. Estima-se que 12 milhões de pessoas em todo o mundo estejam acometidas por doenças transmitidas por alimentos. Este trabalho tem o objetivo de investigar a frequência de enteroparasitoses nos manipuladores de alimentos de escolas públicas de Morrinhos, GO. No período de abril a setembro de 2006, 42 indivíduos com idade entre 19 e 66 anos foram submetidos ao exame coproparasitológico de fezes pelas técnicas de Hoffman e Faust. Foram examinadas amostras de fezes em triplicata de cada indivíduo. A pesquisa realizada foi positiva em 48\% das amostras, E. coli foi o parasita mais frequente $(62,25 \%)$. Com a pesquisa, observamos que os cuidados higiênico-sanitários por parte dos manipuladores de alimentos foram baixos.

Palavras-chave: Saúde pública; Parasitas entéricos; Alimentos.

\begin{abstract}
Illness connect to foods are a serious public health problem. Million of people all around the world are infect by some kind of food sick. This work has as point of investigate the frequency of enteroparasitoses in food handlers in public schools of Morrinhos - GO. In a period of April to September's 2006 peoples of 19 and 66 years old did coprology exam by Hoffman and Faust tecnics. Were investigated three simples of each people. The research was 50\% positive. The most frequency parasite was E. coli parasite, with $81.25 \%$. Was observed with hygienic conditions by food handlers was peccary.
\end{abstract}

Keywords: Health public; Enteric parasites; Foods. 


\section{INTRODUÇÃO}

O Brasil, por sua situação geográfica, condições climáticas e graves problemas sanitários enfrentados porgrande parte da população, apresenta índices de parasitoses elevados. A prevalência das enteroparasitoses varia de acordo com a região e com a população estudada (1). As parasitoses intestinais contribuem para a morbidade e mortalidade de pessoas em todo o mundo, principalmente nos países em desenvolvimento (2).

As parasitoses intestinais assumem um importante papel de saúde pública uma vez que podem ocasionar perdas econômicas pela ausência ao trabalho ou diminuição da produtividade, deficiências no rendimento escolar, gastos com serviços médicos e paramédicos (3). Indivíduos assintomáticos, que pela natureza de seu trabalho estão em contato direto e permanente com alimentos, podem tornar-se fonte potencial de contaminação e disseminação de vários patógenos, entre eles enteroparasitas (4).

A oferta de alimentos isentos de agentes patogênicos os quais possam pôr em risco a saúde do consumidor assumiu mundialmente uma grande relevância em saúde pública (5). As doenças veiculadas por alimentos representam um importante problema de saúde pública, pois se estima que milhões de pessoas de todo o mundo estejam acometidas por doenças transmitidas por alimentos (6), tendo merecido crescente atenção pela elevada frequência e gravidade, tanto nos países desenvolvidos como nos em desenvolvimento (7).

A falta de controle higiênico de alimentos vendidos por manipuladores de alimentos constitui um importante obstáculo para implementar medidas de controle contra as parasitoses intestinais (8). No Brasil, apesar da relevância e da atualidade do problema, são poucos os trabalhos avaliando a ocorrência de enteroparasitoses em manipuladores de alimentos (9).

Muito frequentemente, a falta de cuidado dos manipuladores, como a não obediência às boas práticas e à lavagem incorreta das mãos, propiciam a contaminação dos alimentos (10). O consumo de alimentos contaminados por microrganismos patogênicos pode levar o indivíduo a um quadro infeccioso que varia de um leve desconforto abdominal, a reações severas e até mesmo à morte (11).

Parasitas intestinais, como helmintos de transmissão fecal-oral (Ascaris lumbricoides, Trichuris trichiura e Enterobius vermicularis, Endolimax nana, Entamoeba coli, Hymenolepis nana), podem também estar envolvidos em surtos de doenças transmitidas por alimentos.

Os Ascaris são vermes longos, cilíndricos e com extremidades afiladas, sobretudo na região anterior. Machos e fêmeas apresentam diferenças morfológicas e de tamanho (12). A E. histolytica tem a capacidade de invadir a mucosa intestinal e colonizar nos tecidos do hospedeiro, produzindo aí formas trofozoíticas maiores que as encontradas na cavidade intestinal. Estas amebas grandes einvasivas constituem a forma "magna" de E. Histolytica (13).

Em regiões onde ocorrem cepas patogênicas de Entamoeba histolytica, surtos de disenteria amebiana podem também estar relacionados com alimentos contaminados (14).

A Entamoeba colié um parasito da cavidade intestinal, onde se nutre de bactérias e detritos alimentares, seus trofozoítos e cistos são eliminados com as fezes. Sua distribuição geográfica é mundial (6). A Endolimax nana é uma pequena ameba (a maioria tendo menos de 12 micrometros), que vive nos segmentos cólicos do intestino humano, sem causar nenhum mal (12). O Hymenolepis nana é um parasito conhecido geralmente como "tênia anã" em vista de suas reduzidas dimensões, tendo no escólex um rostro ou prolongamento retrátil, provido geralmente de uma fileira de acúleos (15).

Os objetivos deste estudo foram analisar os hábitos higiênicos dos manipuladores de alimentos correlacionando as parasitoses encontradas no material biológico deles e analisar o aspecto dos lugares onde os manipuladores trabalhavam; observando a limpeza do local, o uso de luvas e toucas durante o preparo e manuseio dos alimentos, a higienização das mãos, a identificação e incidência dos parasitas.

\section{METODOLOGIA}

Foi realizado um trabalho com 42 manipuladores de alimentos de 11 escolas públicas no município de Morrinhos e de um restaurante, com idades entre 29 e 66 anos, do sexo feminino, onde cada indivíduo preencheu uma ficha de identificação com o número de ordem, o nome da escola e dados pessoais do funcionário: nome, bairro, idade, grau de escolaridade e a data da coleta. A ficha continha também questões relacionadas ao hábito de higiene no local de trabalho, sobre a presença ou não de rede de esgoto no bairro onde residem, bem como se já tiveram parasitoses. 
Foi realizada a coleta de material fecal em recipiente de $100 \mathrm{~mL}$, tampa hermética contendo número de identificação, código da instituição, data e hora da coleta. As amostras foram levadas ao laboratório de pesquisa do Departamento de Biologia da Universidade Estadual de Goiás, onde realizou-se as análises coprológicas em triplicata pelos métodos de Faust (16) e Hoffman (17). Pela metodologia de Faust ou de centrífugo-flutuação, dissolveu-se cerca de $5 \mathrm{~g}$ de fezes em $10 \mathrm{ml}$ de água e filtrou-se em gaze dobrada em quatro. O material foi depositado em cúbico cônico de centrífuga e centrifugado à $1500 \mathrm{rpm}$ por 2 minutos. Desprezouse o sobrenadante, foi suspenso novamente em 10 $\mathrm{mL}$ de água, sendo o processo repetido por três vezes. Pelo método de Hoffman foi dissolvido cerca de $10 \mathrm{~g}$ de fezes em $10 \mathrm{~mL}$ de água destilada, foi filtrado em gaze dobrada em quatro e peneira num cálice de sedimentação. $\mathrm{O}$ frasco foi lavado duas vezes, despejando água na gaze. Então o cálice foi completado com água novamente $\mathrm{e}$ homogeneizado com bastão de vidro e deixado em repouso por duas horas. Com uma pipeta tampada, foi tirada uma amostra do fundo do vértice do cálice, destampando a pipeta após imergi-la. Logo após foi colocada na lâmina, adicionada uma gota de lugol e examinada no microscópio.

\section{RESULTADOS}

Obteve-se por análises pelos métodos de Faust e Hoffmann resultados dos parasitas mais frequentes nos manipuladores de alimentos, e avaliação das condições higiênico-sanitárias. No Figura 1 está descrito o resultado de contaminação dos manipuladores de alimentos.

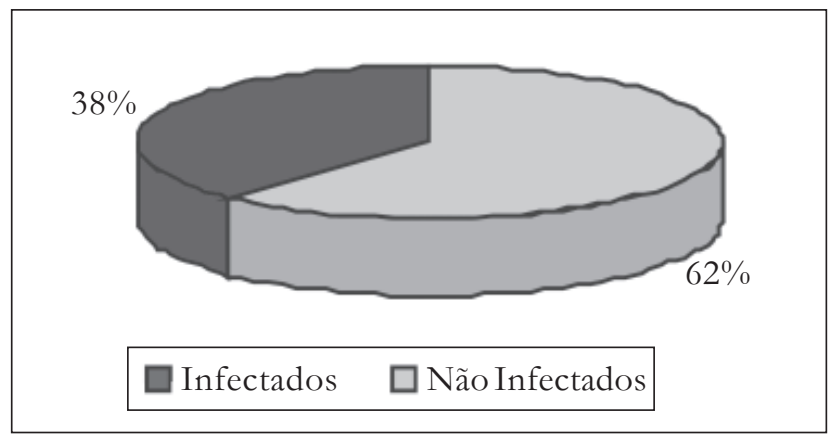

FIGURA 1 - Índice dos manipuladores de alimentos infectados
Observamos na Figura 1 que em 38,1\% dos indivíduos estudados foram identificados um ou mais tipos de parasitos, e que, desses indivíduos, $38 \%$ afirmaram através de questionário nunca terem possuído nenhum tipo de parasitose.

$\mathrm{Na}$ Figura 2 está descrito os parasitas mais frequentes e o percentual encontrado.

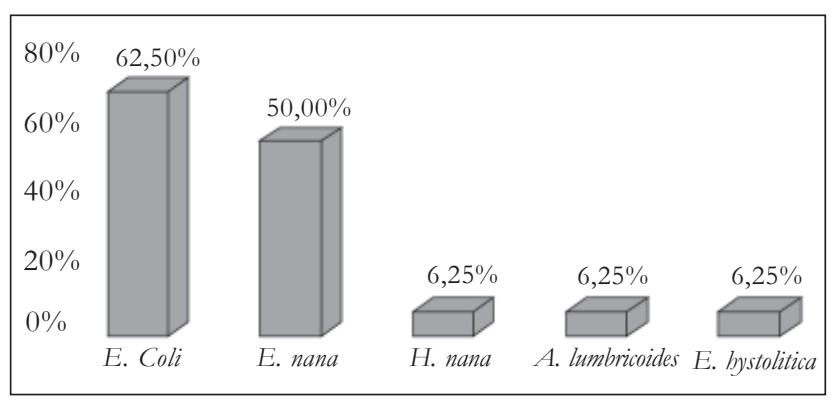

FIGURA 2 - Frequência de parasitas nos indivíduos infectados

Dentre os parasitas encontrados, destacou-se a Entamoeba coli em 62,5\% dos casos, com menor frequência observamos Ascaris lumbricoides (6,25\%); e Endolimax nana com 6,25\% cada (Figura 2).

Observou-se também, por meio de uma análise comparativa, a ocorrência de cada tipo de parasito encontrado pelos dois métodos coprológicos, onde E. histolytica foi evidenciada somente por Faust e a E. nana evidenciada somente pelo método de Hoffmann (dados não mostrados).

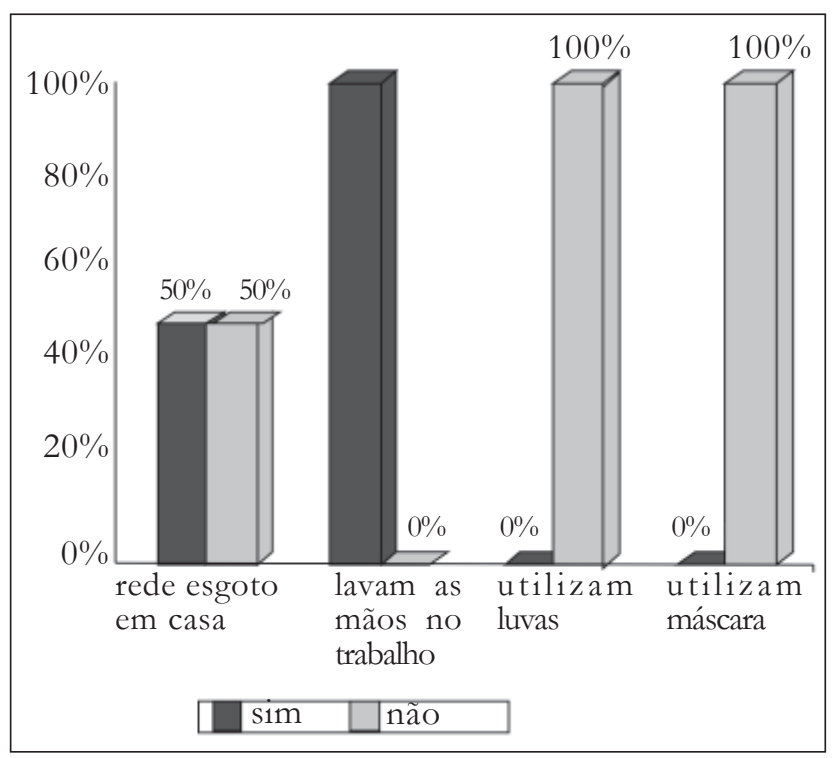

FIGURA 3 - Comparativo entre os manipuladores infectados, rede de esgoto em suas residências e higiene no trabalho 
Constatou-se ainda que, dos indivíduos parasitados, $50 \%$ possuem rede de esgoto em suas residências, porém não se pode afirmar que este fator influencia a presença ou não de parasitos nos manipuladores infectados. Levando em conta os indivíduos parasitados e sua higiene no local de trabalho, todos afirmam lavar suas mãos antes e depois da manipulação dos alimentos, porém o uso de luvas e máscaras durante o processo não ocorre em nenhum caso (dados mostrados na Figura 3).

Analisando os resultados averiguou-se que uma grande porcentagem dos manipuladores de alimentos possui algum tipo de parasitose. Sabendose que as medidas higiênicas no trabalho se encontram insuficientes e que grande parte dos parasitas pode ser transmitida por alimentos contaminados, advertese que há uma necessidade dos responsáveis por este setor e também a realização periódica de análises clínicas para avaliação parasitológica, a fim de que o risco de contaminação dos alimentos seja reduzido ao menor índice possível.

\section{DISCUSSÃO}

Segundo o manual VEDTA (7), parasitas intestinais, como helmintos de transmissão fecaloral (Ascaris lumbricoides, Trichuris trichiura e Enterobius vermicularis), podem também estar envolvidos em surtos de DTA (doenças transmitidas por alimentos). Em regiões onde ocorrem cepas patogênicas de Entamoeba histolytica, surtos de disenteria amebiana podem também estar relacionados com alimentos contaminados; em nosso trabalho, esses parasitas foram identificados com grande prevalência em manipuladores de alimentos.

Quando comparado a estudos feitos com manipuladores de escolas públicas de outras cidades do Brasil, a prevalência de parasitoses em manipuladores de alimentos em nosso trabalho é relativamente proporcional (1). Foi encontrado em crianças de Natal (RN, Brasil) 76,0\% de prevalência. Encontraram em escolares de Salvador (BA, Brasil) $66,1 \%$ de positividade e Marinho et al. (18) encontraram em alunos de Seropédica (RJ, Brasil) uma prevalência total de 33,88\%. Castro et al. (19), analisando pelo método de Hoffman, o coeficiente geral de prevalência de parasitoses intestinais foi de 19,71\%, sendo que Giardia lamblia, Entamoeba coli, Endolimax nana e Ascaris lumbricoides foram os parasitas mais frequentes. Neste trabalho, o parasita mais encontrado foi E. coli, com $62,5 \%$, e o menos frequente foi E. hystolitica, $\operatorname{com} 6,25 \%$.
Nolla e Cantos (9) identificaram resultados de infecção por protozoários significativamente mais expressivos que por helmintos, sendo que o método de Faust foi o mais sensível. Neste trabalho também foi mais expresso a presença de protozoários, encontrando $62,5 \%$ das amostras contaminas com E. coli. Resende et al. (20) identificaram a ocorrência de parasitas intestinais em 17\% (45 casos), 10\% (27 casos) e $10 \%$ (27 casos) para cantineira, servente e serviçal e zelador, respectivamente.

Por outro lado, esses resultados não eliminam a necessidade da adoção de medidas de controle e combate a parasitoses intestinais.

A Vigilância das Doenças Transmitidas por Alimentos está dirigida para a ocorrência de surtos; esses são causados por inúmeros agentes etiológicos e se expressam por um grande elenco de manifestações clínicas, não havendo, portanto, definição de caso preestabelecida. A Notificação ocorre sempre que há evidência epidemiológica de uma fonte comum de água ou alimento originando o surto. A investigação começa identificando comensais, definindo o caso e o período de incubação para elaboração de hipóteses quanto ao agente etiológico e alimento suspeito. A consolidação dos casos permite o cálculo da taxa de ataque, ou seja, o risco a que todos os comensais estão expostos. As medidas de prevenção e controle devem ser tomadas paralelamente à investigação e de acordo com a situação (18).

\section{CONCLUSÃO}

Foiverificada uma porcentagem considerável de parasitoses encontradas nos manipuladores, o que pode levar à diminuição da qualidade dos próprios alimentos, uma vez que esses parasitas poderão ser transmitidos pelos alimentos que os manipuladores infectados preparam. Levando em conta os indivíduos parasitados e sua higiene no local de trabalho, todos afirmam lavarsuas mãos antes e depois da manipulação dos alimentos, porém, o uso de luvas e máscaras durante o trabalho não ocorre em nenhum caso. Também foi verificado que $50 \%$ dos parasitados possuem rede de esgoto em suas residências, apesar de não ser possível afirmar se este fator influencia ou não a presença de parasitas nos infectados.

Analisando os resultados, averiguou-se que uma grande porcentagem dos manipuladores de alimentos possui algum tipo de parasitose. Sabendose que as medidas higiênicas no trabalho se encontram insuficientes e que grande parte dos parasitos pode 
ser transmitida por alimentos contaminados, advertese que há uma necessidade dos responsáveis por este setor, além da realização periódica de análises clínicas para avaliação parasitológica, a fim de que o risco de contaminação dos alimentos sejam reduzidos ao menor índice possível.

\section{REFERÊNCIAS}

1. Saturnino ACRD. Relação entre a ocorrência de parasitas intestinais e sintomatologia observada em crianças de uma comunidade carente de Cidade Nova, em Natal-Rio Grande do Norte, Brasil. Rev Brás Anal Clín. 2003;35(2):85-87.

2. ReyL. Parasitologia: parasitas e doenças parasitárias do homem nas Américas e na África. 2a ed. Rio de Janeiro: Guanabara Koojan; 2001. 156 p.

3. Bloch M. El parasitismo intestinal um tabu que deve de ser destruído. Rev Inst Invest Méd.1982;10(2):102-7.

4. Quick R, Paugh K, Addiss D, Kobayashi J, Baron R. Restaurant-associated outbreak of giardiasis. J Infect Dis. 1992;166(3):673-6.

5. Orlandi PA, Chu DMT, Bier JW, Jackson JG. Parasites and the food supply. Food Tech. 2002;56:72-81.

6. Kaferstein FK, Motarjemi Y, Bettcher DW. Food borne disease control: a transnational challenge. Emerg Infect Dis. 1997;3(4):503-10.

7. Center For Disease Control and Prevention. Diagnosis and management of food borne illnesses. A primer for physicians. MMWR Recomm Rep. 2001;50(RR-2):1-69.

8. Fontes G, Oliveira KKL, Oliveira AKL, Rocha EMM. Influência do tratamento específico na prevalência de enteroparasitoses e esquistossomose mansônica em escolares do município de Barra de Santo Antônio, AL. Rev Soc Brás Med Trop. 2003;36(5):625-8.

9. Nolla AC, Cantos GA. Relação entre a ocorrência de enteroparasitoses em manipuladores de alimentos e aspectos epidemiológicos em Florianópolis, Santa Catarina, Brasil. Cad Saúde Pública. 2005;21(2):641-645.

10. Burton WRG. Engelkirk GP. Microbiologia para ciências da saúde. 2 a ed. Rio de Janeiro: Guanabara Koogan; 1998.
11. Silva JA. As novas perspectivas para o controle sanitário dos alimentos. Rev. Higiene Alimentar. 1999;12(65):19-24.

12. Mead PS, Slutsker L, Dietz V, McCaig LF, Bresee JS, Shapiro C, et al. Food-related illness and death in the United States. Emerg Infect Dis. 1999;5(5):607-25.

13. Rossignol, JF. Parasitic gut infections. Curr Opin Infect Dis. 1998;11(5):597-600.

14. Gatti LL, Santos CV, Lima WP. Relação entre enteroparasitoses, região ungueal e mãos contaminadas, em crianças e adolescentes, da Instituição Filantrópica de Marília. Rev Bras Anal Clin. 1999;31(4):205-6.

15. Neves DP, Melo AL, Genaro O, Linardi PM, editores. Parasitologia humana. 9a ed. São Paulo: Atheneu; 1995.

16. Faust EC, D’Antoni IC, Odon V, Miller MJ, Perez EC, Sawitz W. A critical study of clinical laboratory techniques for the diagnosis of protozoan cysts and helminthes eggs in feces. I. Preliminary communication. Am J Trop Med. 1938;18:169-83.

17. Hoffman WA, Pons JA, Janer S. The sedimentation concentration method in Schistosomiasis mansoni. Puerto Rico J Publ Hlth Trop Med. 1934;9:283-91.

18. Marinho MS, Silva GB, Diele CA, Carvalho JB. Prevalência de enteroparasitoses em escolares da rede pública de Seropédica, município do estado do Rio de Janeiro. Rev Brás Anál Clín. 2002;34(4):195-6.

19. Castro AZ, Viana JCD, Penedo AA, Donatelle DM. Levantamento das parasitoses intestinais em escolares da Rede Pública na cidade de Cachoeiro de Itapemirim ES. Newslab. 2004;64:140-4.

20. Rezende CHA, Costa-Cruz JM, CardosoGennari ML. Enteroparasitoses em manipuladores de alimentos de escolas públicas em Uberlândia (Minas Gerais), Brasil. Rev Panam Salud Publica. 1997;2(6):392-7.

Recebido: 30/06/2007

Received: 06/30/2007

Aprovado: 10/11/2007

Approved: 11/10/2007 